\title{
Assessment of Potential Barriers to the Implementation of an Innovative AB-FB Energy Storage System under a Sustainable Perspective
}

\author{
María Blecua-de-Pedro ${ }^{1}$ (D) and Maryori C. Díaz-Ramírez ${ }^{1,2, *(D)}$ \\ 1 Research Centre for Energy Resources and Consumption (CIRCE), 50018 Zaragoza, Spain; mblecua@fcirce.es \\ 2 Instituto Universitario de Investigación CIRCE, Fundación CIRCE, Universidad de Zaragoza, \\ 50009 Zaragoza, Spain \\ * Correspondence: mdiaz@fcirce.es; Tel.: +34-976-76-22-31
}

check for

updates

Citation: Blecua-de-Pedro, M.; Díaz-Ramírez, M.C. Assessment of

\section{Potential Barriers to the}

Implementation of an Innovative

AB-FB Energy Storage System under a Sustainable Perspective. Sustainability 2021, 13, 11042. https:/doi.org/ $10.3390 /$ su131911042

Academic Editor:

Enrique Rosales-Asensio

Received: 17 July 2021

Accepted: 3 September 2021

Published: 6 October 2021

Publisher's Note: MDPI stays neutral with regard to jurisdictional claims in published maps and institutional affiliations.

Copyright: (c) 2021 by the authors. Licensee MDPI, Basel, Switzerland. This article is an open access article distributed under the terms and conditions of the Creative Commons Attribution (CC BY) license (https:/ / creativecommons.org/licenses/by/ $4.0 /)$.

\begin{abstract}
The novel acid-base flow battery technology stores electrical energy using $\mathrm{pH}$ and salinity gradients in water and is suitable for different capacities (kWh-MWh scale) because of its scalable technology. Due to the flexibility of this system, it is predicted to provide technical, economic, and environmental benefits for supporting renewable energy integration, such as wind and solar energy, within the electricity generation system. However, its level of acceptance might be affected by additional conditioning factors in terms of policies and maintenance. To elucidate the relevance of the possible barriers to the implementation of the innovative AB-FB system, this work introduces an approach based on the analytic hierarchy process developed at three levels of hierarchy under a sustainability perspective. An exhaustive literature review as well as an assessment of experts' evaluations were performed to identify the barriers in terms of technical, economic, environmental, policy, and maintenance aspects. Based on the results, the cost parameters (mostly attributed to the stack cost), followed by technical and environmental criteria, were deemed to be of the highest priority.
\end{abstract}

Keywords: energy storage technologies; acid-base flow battery; barriers; analytic hierarchy process; sustainability

\section{Introduction}

Energy storage systems (ESSs), such as batteries, are challenging and promising tools to overcome the limitations of electricity generation based on solar and wind energy sources [1]. These types of resources involve strong fluctuation and intermittency, hindering proper grid electricity supply [2] and, thus, are an emerging technological challenge to be solved. As a result, ESSs have become a key tool for overcoming the technological challenges related to increasing penetration of renewable and distributed energy resources (DERs). These systems have been proven to help in services such as fast frequency response and energy arbitrage [3]. The benefits of using ESSs for grid balancing have been shown by several techno-economic studies $[4,5]$. In particular, batteries are an attractive option to be incorporated into low-carbon distributed power system planning [6,7].

An innovative ESS that can be used for overcoming the intermittency of renewable resources is the so-called acid-base flow battery (AB-FB), which is appropriate to this renewable energy support (RES) scenario.

This innovative technology is composed of an electrolytic solution, with water and salt as the main components. The AB-FB charges by converting electrical energy into salinity gradients when $\mathrm{Na}^{+}$and $\mathrm{Cl}^{-}$ions from the salt solution $(\mathrm{NaCl})$ migrate through the ion exchange membranes to be separated into three different solutions: freshwater, $\mathrm{NaOH}$, and $\mathrm{HCl}$. This mechanism is well-known as acid and base generation from salt solutions by electrodialysis with bipolar membranes [8]. During the discharge, electrical energy can 
be recovered from the salinity gradient by neutralising the acid and base solutions at the bipolar junction inside the bipolar membrane to form water. During charge and discharge reactions, the $\mathrm{Fe}^{3+} / \mathrm{Fe}^{2+}$ redox pair reactions occur in the positive and negative electrodes, feeding the external circuit with electrons. A detailed description of the AB-FB system can be found elsewhere [9-11].

Based on the specific characteristics of this new technology, such as its flexibility and scalability [9-11], it is thought to provide potential technical, economic, and environmental benefits to grid balancing. These potential impacts have been elucidated in a comparative analysis recently performed on the AB-FB and vanadium redox flow battery (VRFB) systems [12]. This work performed two life-cycle studies using a cradle-to-grave approach by assessing data gathered by both systems. On the one hand, the environmental impacts of the two systems were compared and calculated based on the ReCiPe midpoint method and SimaPro software. On the other hand, the economic evaluation of the two systems suggested the assessment of the total capital cost and the life-cycle cost.

According to earlier results [12], the new battery system could be a more competitive option compared with the VRFB system. One of the main advantages of the AB-FB system is that its main component is water, which represents $98.7 \%$ of the total system composition. This characteristic positively influences the electrolyte environmental implications in contrast to the vanadium-based electrolyte, whose main component, vanadium, is listed as a critical raw material [13]. Impacts related to the vanadium-based electrolyte of the VRFB have been extensively reported in the literature as the main constraint on its environmental performance in the manufacturing stage [14,15]. Regarding the economic aspects, the AB-FB system has also been recognised as providing competitive costs compared with the VRFB system. The higher investment cost of the VRFB system is mostly influenced by the energy subsystem and, in particular, the vanadium-based electrolyte cost. Power subsystem costs seems to be very similar for both ESSs [12], mostly attributed to similarities in terms of the stack cost, which is mainly made of membranes [11].

Due to these advantages, AB-FB technology can become a potential competitor of VRFB, especially in terms of its environmental and economic aspects. Nevertheless, there are other aspects that should be evaluated to implement this ESS under a sustainability perspective. Together with the expected benefits in terms of technical, economic, and environmental aspects, the implementation and final acceptance of an ESS might also depend on legislation- and maintenance-related issues. Accordingly, the acceptance level of new technology is expected to be affected by a multivariable process. For this reason, a detailed assessment of the conditioning factors in attaining technology acceptance are required. With this approach, the most influencing factors can be detected and assessed.

In this vein, a multicriteria decision-making process (MCDM), in particular, the analytical hierarchy process (AHP), constitutes one powerful approach that is mostly applied to sustainable energy projects [16-18]. The AHP is an efficient decision-making tool that assesses the conditioning factors through an implementation of the system. Klein [19] applied an MCDA to assess the technical, economic, and environmental implications of sustainable thermal ESSs and also explored policy issues. Özkan et al. [20] also conducted a political and social impact assessment. This method also allows one to quantify the level of influence of each conditioning factor on making the final decision for the implementation of the new system.

This research proposes a systematic method based on the AHP model to carry out an advanced evaluation of the relevant aspects to be considered for the acceptance of new AB-FB technology. As such, the first objective of this work was to perform a detailed assessment of five key influencing parameters: costs, environmental impacts, technological aspects, policies, and maintenance issues. This assessment was focused on elucidating the specific barriers to implementing AB-FB technology under a sustainability perspective. After completing this step, the second objective of this study was to analyse the relevance of each parameter and its corresponding barriers through AB-FB system implementation. 
Accordingly, the results of this study provide an overview of the main constraints related to the AB-FB acceptance level. Based on these results, guidelines for overcoming these barriers are also generated that can be considered by designers, technicians, and industries to overcome the potential constraints of similar technologies. Finally, this research also helps technology developers to easily identify and prioritise barriers while optimising system benefits.

\section{Materials and Methods}

As previously mentioned, there are different factors that might influence AB-FB technology implementation from a sustainability perspective and acceptance within the RES scenario. Among them, special attention was given in this research to five key influencing parameters: costs, environmental impacts, technological aspects, policy, and maintenance issues. The methodology developed in this research was focused on quantifying the relevance of these parameters and their specific barriers. Moreover, the identification of actions to overcome related limitations was studied as well. To carry out a comprehensive analysis of the abovementioned aspects, an innovative approach based on the AHP method was applied. Under this premise, three main stages were developed:

(i) To identify specific barriers corresponding to each of the five key influencing parameters (called criteria, according to the AHP model): technical, economic, environmental, policy and maintenance aspects.

(ii) To define specific recommendations to overcome the detected specific barriers (called sub-criteria, according to the AHP model).

(iii) To quantify the influence of the five parameters (criteria) and their specific barriers (sub-criteria) using the AHP method.

In the following sections, specific actions to carry out each stage are presented. An overview of the methodological approach based on the three stages is depicted in Figure 1.

Potential barriers to the implementation of the AB-FB system
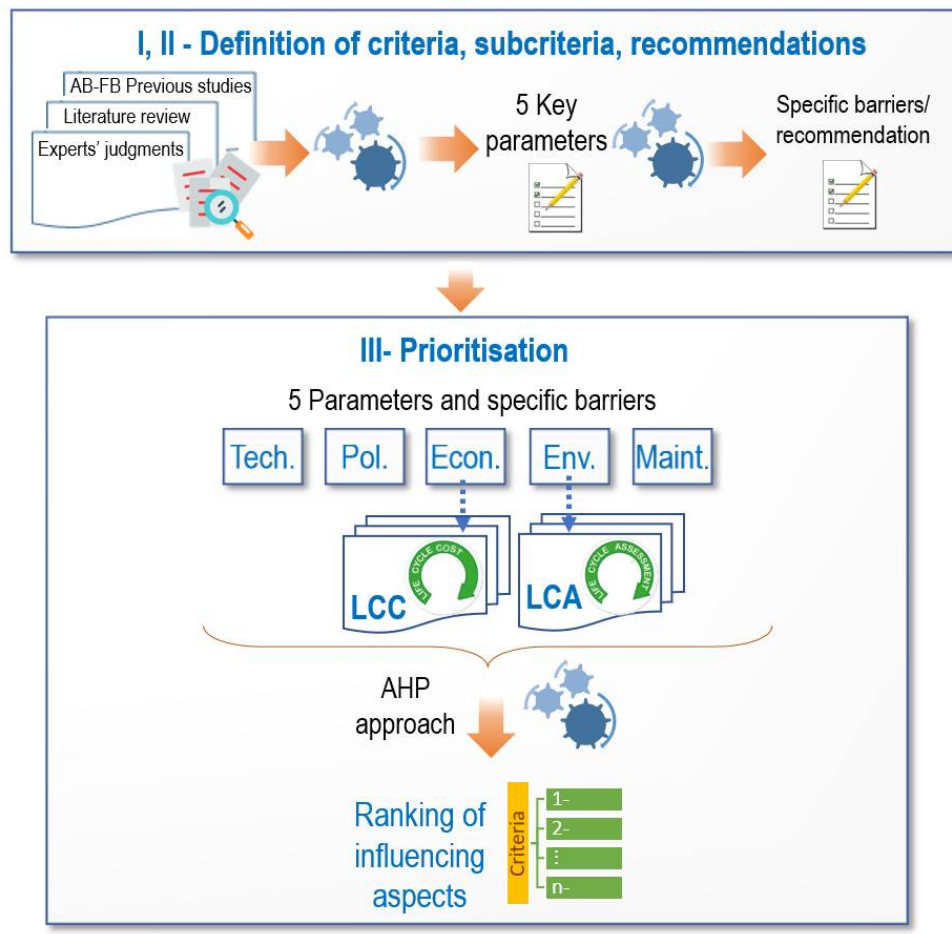

Figure 1. Overview of the methodological approach. 


\subsection{Definition of the Key Influencing Parameters and Related Specific Barriers under a Sustainability Perspective}

Sustainability is about the balance or harmony among the economic, social, and environmental sustainability pillars and the relationships between them: economic-social, social-environmental and environmental-economic aspects, as can be seen in Figure 2. In this work, the selected criteria, or the main influencing factors, exhibit a close relation to the three sustainability pillars and their interrelations. In this way, technical (C1) and maintenance (C4) criteria are related to the existing interrelations among the three main pillar categories, in contrast to the environmental (C3) and economic (C2) criteria (see Figure 2).

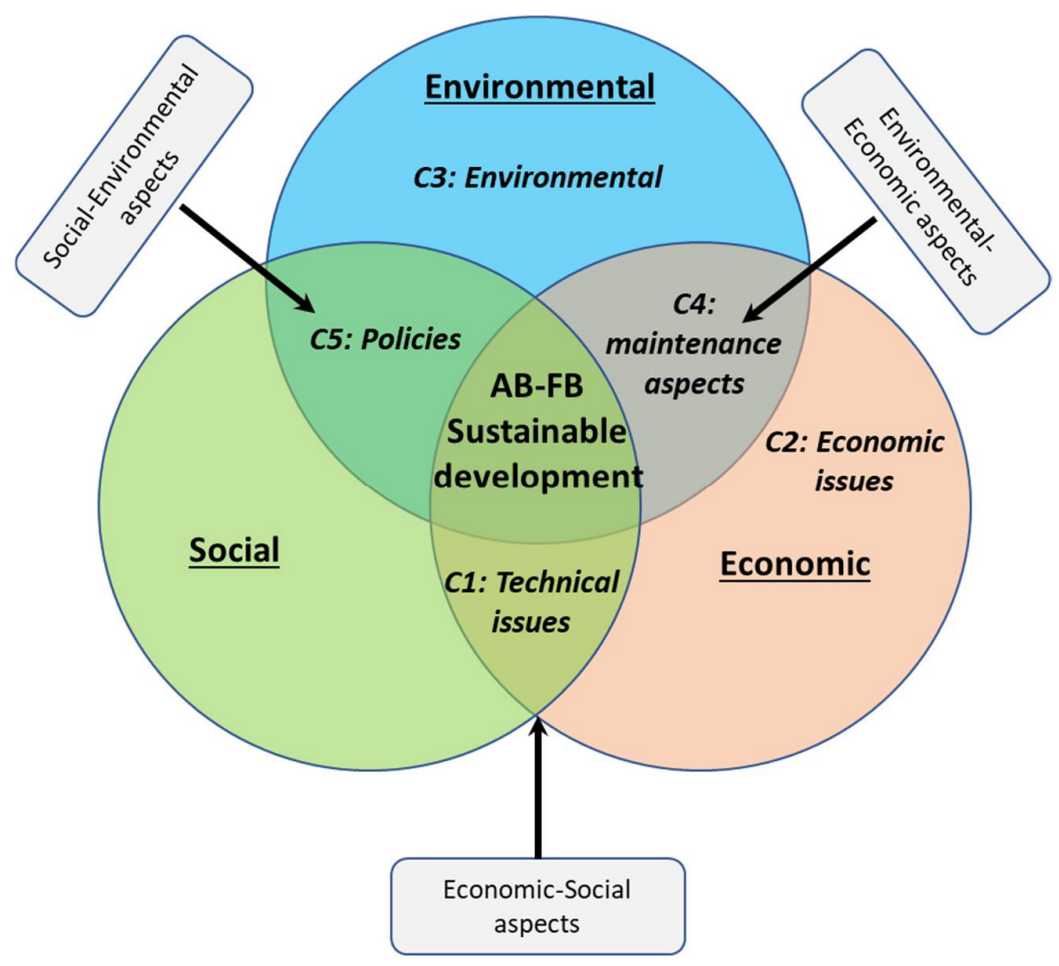

Figure 2. Criteria distribution under a sustainability framework.

Concerning the policies criterion (C5), it is well-known that each pillar is supported by corporate policies, procedures, and guidelines to help in the management of material sustainability issues. Nevertheless, as ESS implementation policies exhibit a social and environmental character, it was proposed that $C 5$ correspond to the social-environmental aspects. Accordingly, the five criteria are represented by one of the three sustainability pillars and their interrelations, which confirmed that all of them are relevant to the assessment of AB-FB technology implementation.

To provide a deeper knowledge of the implications of each criterion, a detailed revision of these five parameters was carried out. First, an extensive literature review was carried out to identify the specific barriers. By this action, it was possible to elucidate specific conditioning factors (barriers) related to each considered aspect (costs, environmental impacts, technological aspects, policies, and maintenance). Social barriers were not analysed in this study. Information related to the acceptance of other technologies, such as the VRFB, was also revised. Second, the preliminary list of selected factors was further complemented by results from previous research works related to the AB-FB system, specifically those from the environmental and economic analysis of the AB-FB system [12]. Third, meetings with experts involved in the development of the new AB-FB technology were held. The panel of experts included industrial participants with recognised backgrounds as a membrane manufacturer, a technology provider, as well as researchers and academics with expertise in the field of ESS, sustainability and decision support methodologies. The number of 
participants was properly decided since the AHP is not a statistical tool, and, accordingly, there is no rule that defines the number of participants. In particular, the panel of experts was formed to attain a deeper assessment of the detected barriers identified by the literature review as well as to provide guidelines for overcoming the barriers. Actions performed by the panellists comprised communication via mail, questionnaires, and a workshop. The background diversity and number of panellists were aimed at guaranteeing an objective survey process.

The main outcome of the expert panel meeting was information concerning the implications of the detected barriers, subsequent discussions, and the definition of recommendations to overcome these barriers. Specific information concerning the identified barriers and recommendations is presented in Section 3.1.

\subsection{Prioritisation of Barriers}

All the information gathered in the previous step corresponded to the criteria and sub-criteria factors influencing the implementation of the new technology. They were further analysed through a methodological approach based on the AHP model.

The AHP is a structured technique developed by Thomas L. Saaty in the 1970s and used to make complex decisions. This technique has been demonstrated to be an accurate approach to quantifying the weights of various decision criteria $[18,20,21]$. Within a set of several options organised in the same domain, each collaborator compares the relative importance of each pair of items using a specific questionnaire. Specifically, the AHP model offers not only a simple, but also a complete evaluation method that can facilitate the decision-process for businesses and public sector institutions.

The model applied in this work involved three levels of hierarchy (see Figure 3). The first level corresponded to the goal of the study problem (goal level). In this case, it was technology implementation and acceptance. The second level presented the main influencing parameters (criteria level). In this case, it corresponded to costs, environmental impacts, technological aspects, policies, and maintenance issues. The third level of the hierarchy (sub-criteria level) presented the specific barriers related to each criterion. Each of the main criteria involved 3 to 6 barriers.

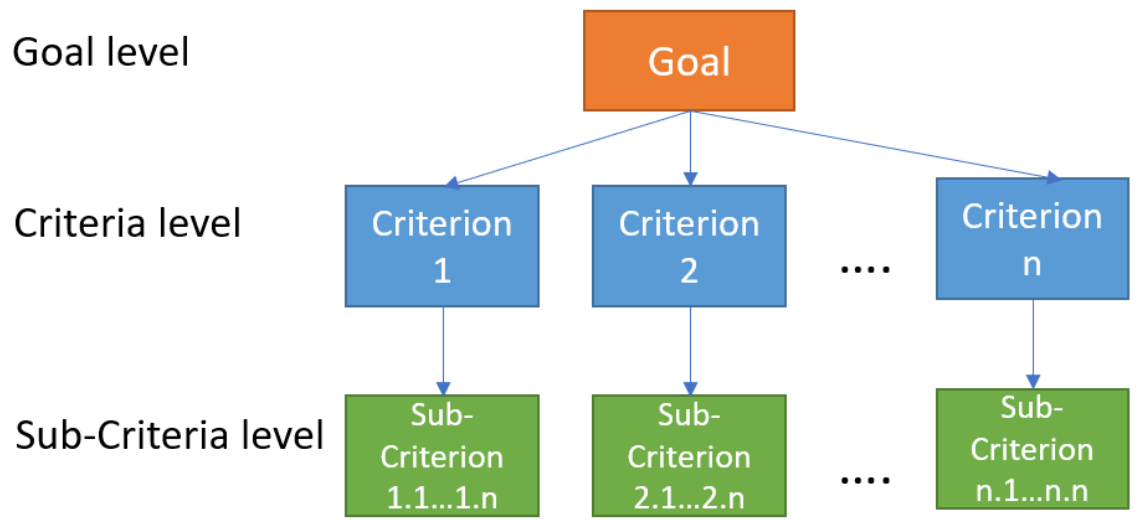

Figure 3. Scheme of the hierarchy structure.

In the AHP analysis, expert opinion and judgement are important steps in the decisionmaking process. For this purpose, a special questionnaire was answered by the expert panel participants. Comparisons were made by prioritising the 5 criteria and the sub-criteria within the same criteria. In this manner, two levels of comparison were carried out: one for ranking the parameters (criteria level) and another one for ranking the barriers (sub-criteria level). Keeping with the AHP method, scores were normalised according to the required punctuation system, i.e., Saaty's scales, range 1-9 (see Table 1). 
Table 1. Saaty's scale values for pairwise comparison.

\begin{tabular}{|c|c|}
\hline Punctuation & Punctuation Definition \\
\hline 1 & Equal importance of the row criterion over the column criterion \\
\hline 2 & $\begin{array}{l}\text { Between equal and weak importance of the row criterion over the } \\
\text { column criterion }\end{array}$ \\
\hline 3 & Weak importance of the row criterion over the column criterion \\
\hline 4 & $\begin{array}{l}\text { Between weak and strong importance of the row criterion over the } \\
\text { column criterion }\end{array}$ \\
\hline 5 & Strong importance of the row criterion over the column criterion \\
\hline 6 & $\begin{array}{l}\text { Between strong and demonstrated importance of the row criterion over the } \\
\text { column criterion }\end{array}$ \\
\hline 7 & Demonstrated importance of the row criterion over the column criterion \\
\hline 8 & $\begin{array}{l}\text { Between demonstrated and absolute importance of the row criterion over the } \\
\text { column criterion }\end{array}$ \\
\hline 9 & Absolute importance of the row criterion over the column criterion \\
\hline
\end{tabular}

To facilitate the calculations related to the overall priorities of criteria and the local priorities of sub-criteria, an AHP free online Excel template by Klaus D. Goepel [1] was employed. In this template, first, the individual judgements of the experts based on Saaty's scales of 9 points were entered, and second, the individual priorities and level of consistency ( $C R$ index) were generated. The $C R$ index was necessary to check the consistency of judgements and preferences. Based on Saaty's data, a consistency ratio of 0.1 (i.e., 10\%) or less was required to continue the AHP analysis. On the other hand, if the CR index was higher than 0.1, the pairwise comparison evaluation procedure would be repeated to improve the consistency. Then, the derived overall priorities for the domains and the local priorities for the barriers within each domain were generated. Finally, aggregation of the individual judgements was calculated to attain the overall weight of each sub-criterion. The results are included in Section 3.2.

\section{Results and Discussions}

\subsection{Barrier Categories and Recommendations}

According to the methodological process described in Section 2.1, different specific barriers were identified concerning each of the five key parameters: costs, environmental impacts, technological aspects, policies, and maintenance issues. For all of them, different recommendations to overcome possible limitations concerning the new technology acceptance and implementations were also explored. The identified barriers are grouped in Table 2. An overview of each of the detected barriers corresponding to each parameter is presented in the following sections.

\subsubsection{Technical}

Technical barriers are those related to the chemistry and design of the battery, which determine its voltage, capacity, power, energy density, and application. Concerning this parameter, six barriers were identified.

\section{- Low specific energy of the new system:}

The specific energy of a battery system is related to the energy provided by the battery divided by the weight of the whole system. The specific energy of the AB-FB system was calculated as $3.25 \mathrm{Wh} \mathrm{kg}^{-1}$, which was six times lower than the value calculated for the VRFB system, $19.4 \mathrm{Wh} \mathrm{kg}^{-1}$. These values came from the nominal capacity of the batteries (9.6 MWh for AB-FB and 8.3 MWh for VRFB) and the total weights of the battery systems (1,961,491 kg for AB-FB and 304,809 kg for VRFB) [12]. Differences between the specific energy values mostly referred to the amount of electrolytes needed by each 
battery. Vanadium technology uses less electrolytes because of the higher energy density of the transition metal electrolytes (vanadium-based electrolyte). Consequently, this type of electrolyte makes the VRFB much less sustainable than the AB-FB technology (water-based). Furthermore, the AB-FB system is expected to reach specific energies closer to $11 \mathrm{Wh} \mathrm{kg}^{-1}$, which would lessen the difference with the VRFB [11,22,23].

Optimisation of the operating and design variables as well as the membranes is required for improving the specific energy density, although the optimisation window is small, even in the long term. To overcome this limitation, we foresee that AB-FB technology applications will be limited to a specific ion concentration in the electrolyte that will help them attain an increase in the specific energy density.

- Low energy efficiency and corresponding energy losses

Round-trip efficiency (RTE) is the percentage of electricity put into storage that is later retrieved. The higher the round-trip efficiency, the less energy is lost in the storage process. The AB-FB RTE is equal to $65 \%$, which is lower than the VRFB (75\%) [12], although it seems to be higher than the one reached using hydrogen as energy storage vector [24,25]. It is expected that the AB-FB RTE will reach values higher than $70 \%$ (based on preliminary and not-yet-published research studies).

This type of limitation is expected to be addressed by optimising operating conditions, such as charge and discharge cycles according to the application, or improving the stack design. Improvement of membrane selectivity might also increase the AB-FB RTE. Nevertheless, it is a challenging condition to be overcome in the long-term period.

- Need for a high number of stacks due to low battery reversible voltage

A total of 36 stacks are needed for reaching a power equal to $1 \mathrm{MW}$, whereas the VRFB needs only two stacks [12]. This issue mainly occurs because of the low reversible voltage of the chemical reaction that occurs inside the power subsystem of the new AB-FB system. The use of a high number of stacks also has a negative impact on manufacturing costs. It is expected that the optimisation of the manufacturing process will lead to the production of small power units that reduce the fabrication costs and improve the total capacity of the battery. This type of modular system will also facilitate the maintenance of the battery.

- Lack of information regarding the battery system lifespan and component replacement time

Due to the newness of the AB-FB system, there is a lack of knowledge regarding the lifespan of its components. Based on the literature [26], we estimated 10 years of lifespan for the power subsystem components and a 20-year battery lifespan.

Available data on bipolar membrane electrodialysis (BMED) units suggested a lifespan of about 3-5 years [27-29]. In the case of the membranes, their lifespan depends on the concentration during the charging process. Higher concentration lowers the life of the membranes, but the lifespan also depends on the membranes' manufacturer and the working temperature. As an approximation, in the case of this AB-FB, the membranes' lifespan was considered to be 10 years, owing to the moderate temperature (below $30^{\circ} \mathrm{C}$ ) and the use of acid and base solutions that are not oxidative because of their low concentrations (below $1 \mathrm{M}$ ).

The best approach for overcoming this barrier would be running ageing experiments on materials and stacks in the AB-FB system as well on available commercial membranes.

- Low number of battery cycles before regeneration of the electrolyte solutions

After long operation times or if there is a leakage in the system, co-ion migration, diffusion of the species, and water fluxes may cause a variation in the electrolyte composition and concentrations.

Regeneration of the electrolyte at the proper solution concentration in each tank might be useful to overcome this effect. This process consists of mixing up all the solutions and separating them properly in their corresponding tanks.

During regeneration, loss of iron might occur, which is one component of the electrolyte rinse solution. This is the most important issue because of its negative impact on 
the efficiency of the battery and, consequently, on the battery power. This limitation can be overcome by optimisation of the tank regeneration process and the use of bigger iron molecules despite its effect on the sustainability of the battery.

- Finding the right application according to the location of the battery

The AB-FB system requires a high amount of electrolytes and, therefore, the need for big facilities for locating the reservoirs. In addition, because it needs a large amount of water, the battery application should be linked to the battery location. Depending on the battery capacity, large areas are needed for the installation of the system.

Proposed recommendations can be found below:

1-Personalised structure of the battery for each location.

2-Possibility of decoupling the battery components (stack and tanks) location and transferring the electrolytes to the stack.

3-Combining desalination plant locations, leading to a brine valorisation process.

Table 2. Parameters and specific barriers to the technology acceptance of the AB-FB system.

\begin{tabular}{cl}
\hline Parameters & \\
\hline & Low specific energy \\
& Low energy efficiency \\
& High number of stacks \\
& Unknown battery system lifespan \\
& Low number of battery cycles before regeneration \\
& Right application \\
& Membrane cost \\
& High stack assembly cost \\
& High PVC bag and spacer + gasket costs \\
& Electrolyte production and storage \\
\hline \multirow{3}{*}{ C2: Economic } & Challenging materials \\
& Water footprint \\
& Environmental burden from wind and PV installations \\
& Pump replacement time \\
& Quality of the water \\
& Leakage problems \\
& Sensor calibration (BMS maintenance) \\
& Stack replacement (10 years) \\
& Definition of BESS (Battery Energy Storage Systems) \\
& Ownership of the BESS \\
& Differences in grid codes \\
& Non-inclusive grid codes \\
& Double taxation \\
\hline
\end{tabular}

\subsubsection{Economic}

Economic barriers are the ones related to the cost of the system at the different life stages: production, installation, operation, and disposal. Information was provided by a life-cycle cost analysis of the new AB-FB system [12].

- Membrane cost dominates the stack cost

It was identified that $65 \%$ of the stack costs were for the membranes, which means that the stack cost is too dependent on the membrane cost. Factors influencing the membrane cost include the following:

1-Expensive chemicals are used during membrane production to reach more robustness.

2-The production of the bipolar membrane is complicated and expensive, and its market share is still limited. There are just a few suppliers on the market.

3-In comparison with the VRFB, the AB-FB system has three bipolar membranes in each cell while the VRFB has only one. 
It is expected that increasing the manufacturing of membranes at a commercial scale will minimise membrane production costs. However, this implies a long-term perspective.

- High expenditure on stack assembly

The AB-FB system has a high stack assembly cost because of the large number of cells needed for a $1 \mathrm{MW} / 9.7 \mathrm{MWh} \mathrm{BAoBaB}$ system, owing to the low power density values. In total, 8064 cells are needed, in comparison to 155 cells for a VRFB system with the same technical characteristics.

As mentioned in the technical barriers, the production of repetitive units, for example, a $1 \mathrm{MW}$ stack production, would lead to lower production costs, and if the process is automated, the assembly cost can be extremely reduced. Furthermore, this would lead to an easier replacement of the stack unit, improving the maintenance of the battery.

- High investment cost of PVC bags, spacers, and gaskets

The cost of the PVC bags for storing the electrolytes accounts for approximately $70 \%$ of the cost for the energy system. The cost of the PCV bags is around EUR $29 \mathrm{k} / \mathrm{MWh}$. However, it is important to consider that the energy subsystem is mainly comprised of the electrolyte, which is water, and consequently, it is cheaper compared to the VRFB system (EUR $40 \mathrm{k} / \mathrm{MWh}$ vs. EUR $149 \mathrm{k} / \mathrm{MWh}$ for VRFB). In the same way the spacers and gaskets accounted for $16 \%$ of the stack cost, but this was not a big issue, as the biggest concern related to the stack cost was the membranes cost.

To reduce the cost of the PCV bags several options can be taken, such as the use of regular and standardised containers, but this option will result in larger space requirements. In the case of the spacers and the gaskets, the cost will be reduced if an automated production process is used. Accordingly, this limitation should be addressed in the future.

- Large facilities needed for storing $B A o B a B$ electrolyte

Due to the low energy density of BAoBaB large volumes of electrolyte are needed, which has implications in terms of storage and the use of water as a resource. On the one hand, large areas are required for installation of the system. On the other hand, the water demand means that location will also be limited to water availability.

To overcome this limitation, the implementation site should be analysed and possibly related to areas where the use of water is not a main constraint_-for instance, areas with good availability of this resource or places where it is easy to transport the water in trucks.

\subsubsection{Environmental}

Environmental barriers are the ones related to this technology's contribution to the pollution of the planet during its different life stages: production, installation, operation, and disposal. The environmental behaviour of the AB-FB system was analysed by performing a life-cycle analysis (LCA) of the system from a cradle-to-grave perspective [12].

- Challenging materials

Challenging materials were identified during the LCA, and they referred to those materials that contribute to the highest environmental impacts. In the case of BAoBaB technology, these materials were found in the power and peripheral components:

1 - Steel used in the frame and the inverter.

2-Copper used in the electrodes and inverter.

3-Polyethylene (PE) and polyvinylchloride (PVC) used for producing the gaskets.

4-Sodium nitrate and aniline used for the membrane production.

Considering that the use of specific materials cannot be avoided and focusing on a sustainability perspective, some actions can be defined to minimise the environmental burden attributed to the consumption of some of the resources.

In the case of the steel used for the frames, gluing of the membranes and spacers, might reduce the necessity of heavy metal frame. Regarding plastic materials, PE and PVC, it might be difficult to replace them by other more environmentally benign plastics due to their limitations to support regarding acid and basic AB-FB electrolyte components. 
Regarding membranes, a clarification should be considered. Due to confidentiality issues, the exact composition of the membranes currently developed for the new battery technology could not be modelled in the LCA presented in a previous study [12]. Accordingly, the inventory was approximated with the (s)peek data provided by Weber et al.

The environmental impacts attributed to the materials required for the (s)peek membrane production are specifically related to the use of sodium nitrate and aniline. They are the materials that contributed most to the carbon footprint, so their replacement by more environmentally benign materials in the future should be assessed.

Finally, besides investigating the options for substituting the required materials with others having a lower environmental burden, actions might also be focused on evaluating different circular economy approaches such as recyclability. This would lead to lower environmental impacts, so more attention should be paid to this type of approach. Nevertheless, information concerning recyclable battery approaches is still limited in the literature.

\section{- High-water footprint}

Water content in the AB-FB system is almost $98.7 \%$ weight percent of the battery; therefore, a high-water footprint is expected to be attained with the new technology. A possible measure to minimise this indicator's impacts might be using the new technology close to areas without availability problems.

\section{- Environmental burden from wind and PV installations}

Although this constraint is not directly related to the AB-FB technology, the whole system implies the use of renewable energy as a more sustainable way to generate and store energy. As the limitations are mostly related to the construction of wind and PV installations, impacts can be minimised by adopting a more sustainable manufacturing process for wind turbines and solar panels.

\subsubsection{Maintenance}

Maintenance barriers refer to maintenance activities related to the AB-FB technology.

\section{- $\quad$ Pump replacement}

Pumps are used for transferring the electrolyte from the PCV bags to the stacks where the chemical reaction takes place. The replacement time has been estimated as every 5 years, based on scenarios defined by Weber et al. [26] for the VRFB system. As the electrolyte used for the VRFB is based on a sulfuric acid solution, more severe damage is expected compared to the water, base, and acid solutions used in the AB-FB technology. Furthermore, the damage to the pumps also depends on the charge and discharge rates, and therefore on the battery application. Switching the pumps on and off frequently may affect their replacement time.

We expect these limitations to be overcome by optimising the process conditions or by using closed-loop or open-loop modes. Additionally, pumps are used in many applications, such as water heating systems in households. However, the replacement of the pumps is normally carried out in production processes when the battery lifespan is reached, so this should not be regarded as an important constraint.

\section{- Quality of the water}

Water is the main component of the AB-FB electrolyte, and because of that fact exhaustive attention must be paid to its maintenance.

On the one hand, the growth of microbial agents is facilitated when the water is exposed to light. On the other hand, the exposure of the water to air could result in the dissolution of the carbon dioxide from the air and the formation of carbonates.

To overcome these limitations, storage units can be designed to avoid light exposure, and, if this is not possible, the addition of a small amount of antimicrobial agent should work. Furthermore, if the tank is designed to be airtight formation of carbonates as well as algae growth might be limited. 
In addition, high perm-selectivity of all membranes and low water permeability of the monopolar membranes will also contribute to reducing the contamination of the water. Accordingly, water maintenance should not exhibit a high cost. Improvements should be focused on the tank design and location, although better membrane selectivity would also help.

- Leakage problems in the system

This is a common problem for the flow batteries, but in this case, because of the nature of the $\mathrm{AB}-\mathrm{FB}$ electrolyte (low acid and base concentrations), a leakage problem in the AB-FB system would be safer than one in the VRFB system (acid electrolyte based on sulfuric acid).

Leakages can be found in the entire AB-FB system, as the electrolyte passes through all of it. However, attention should be especially paid to the PVC bags and the stack. Leakage problems may come from a poor sealing of the hydraulic components. In this case, improvements in the sealing of the battery components will reduce leakages. Gluing/welding of membranes and spacers (already proposed for the reduction in production costs) is another option. In addition to these issues, leakages can also damage buildings and other equipment. Therefore, it is better to place the unit susceptible to leakages in a separate room with special treatment protection of the expected leakage components. The use of the battery management system (BMS) for monitoring and controlling the level of the tanks will also contribute to minimising the effects related to this specific barrier.

\section{- $\quad$ BMS maintenance}

The BMS controls the sensors distributed throughout the whole system. Sensors available for monitoring and controlling should be reliable or at least not provide false alarms or bad operations. In this way, a periodic sensor calibration may be necessary to prevent wrong measurements. A maintenance plan should be carried out to prevent these problems. This maintenance plan should include not only the standard calibration of the different sensors, but also a periodic check protocol that combines sensor data for cross-validation (e.g., voltage and conductivity).

- Stack replacement every 10 years

In this case, it was assumed that the stack should be replaced after 10 years of operation, based on scenarios defined by Weber et al. [26] for the VRFB system. This assumption was due to the lack of knowledge regarding the lifespan of the membranes developed for the AB-FB technology, which are the most critical components of the stack. The stack replacement implies an expenditure of EUR 479k for the membranes alone (65\% of the total stack cost), which is a high maintenance cost.

The running of ageing experiments on materials and stacks will provide more specific information regarding their lifespan. In addition, we expect that membrane production at a commercial scale (for instance, production for an industrial $1 \mathrm{MW}$ unit scale) will reduce these costs and replacement difficulties based on the future availability of producer competitors.

\subsubsection{Policies}

This section explores the barriers related to the course of action proposed or adopted by an organisation or group of individuals to achieve rational goals concerning the integration of ESSs into the electric power grid. As these types of barriers are more related to the EU directrices, solutions are expected to be generated over a long term. Accordingly, a brief explanation of the detected barriers is presented without exploring long-term solutions.

\section{- Definition of BESS}

A clear definition of the BESS by the EU has only been introduced recently. The lack of a common legislation at the EU level has resulted in important differences among EU countries. Due to the lack of a clear definition, ESSs have been often considered as generation units, falling under network codes for generation facilities. 


\section{- $\quad$ Ownership of the BESS}

In most EU countries, it is currently not clear if transmission system operators (TSOs) and distribution system operators (DSOs) can directly operate BESSs for grid balancing. Some countries impose specific limitations.

- Differences in grid codes

Different countries with different grid codes (requirements for the generators; voltage, voltage support) generate inconsistency in the management of the same technologies in the EU.

\section{- $\quad$ Not-inclusive grid codes}

Grid codes in some countries refer only to lead-acid batteries or lithium-ion batteries. The web applications for registering new BESS connections do not include other kinds of batteries.

\section{- Double taxation}

There is a double application of network charges (as a generator and as a load) in some countries (Austria, Germany, Finland, and The Netherlands) that might limit BESS implementation in these locations.

\subsection{Assessment of Barriers' Influence}

As described in Section 3.1, different domains and the barriers related to each domain were identified as key aspects to be considered for AB-FB system implementation. As a result of this analysis, five different domains (technical, economic, environmental, maintenance, and policies) were selected.

The level of possible influence and the relevance of each parameter concerning AB-FB system implementation were assessed by applying the AHP methodology, as indicated in Section 2.2. In this vein, the five domains were subjected to pairwise comparison. Based on the weights assigned to each criterion by the experts, their priority was calculated. The consistency ratio was found to be 0.004 , which was acceptable since it was less than the 0.1 proposed by the AHP method.

Figure 4 shows the priorities of the main criteria. The economic and technical criteria were ranked higher than the other criteria, in that order. In particular, the main concern of the experts regarding $\mathrm{AB}-\mathrm{FB}$ system implementation was the economic aspect. On the other hand, policy issues had the least weight (5\%). Finally, environmental impacts showed results similar to maintenance issues, 14 and 13\%, respectively. Environmental impacts occupied the third position in the ranking, which indicated their relevance to the implementation of this sustainable system.

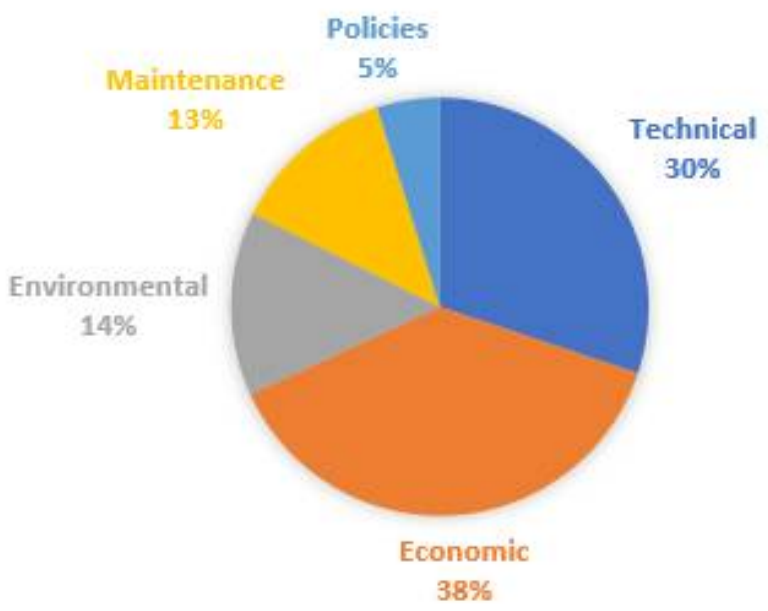

Figure 4. Overall priorities of the main criteria with respect to the goal. 
The economic and technical criteria have been considered very relevant to ESS in the literature [20,30]. Specifically, Escalante et al. [31] focused on developing a new approach to assessing the technical and economic feasibility of flow batteries in remote applications. Other ESSs, such as pumped storage [32,33] or compressed-air energy storage systems [34], were also modelled by considering a techno-economic perspective.

A deeper analysis of the relevance of these five criteria was elucidated by prioritising the sub-criteria level, i.e., the barriers corresponding to each domain. Accordingly, five pairwise comparison matrices with respect to each main criterion were developed based on the experts' opinions. The results for each criterion and corresponding domain are presented in Figure 5.

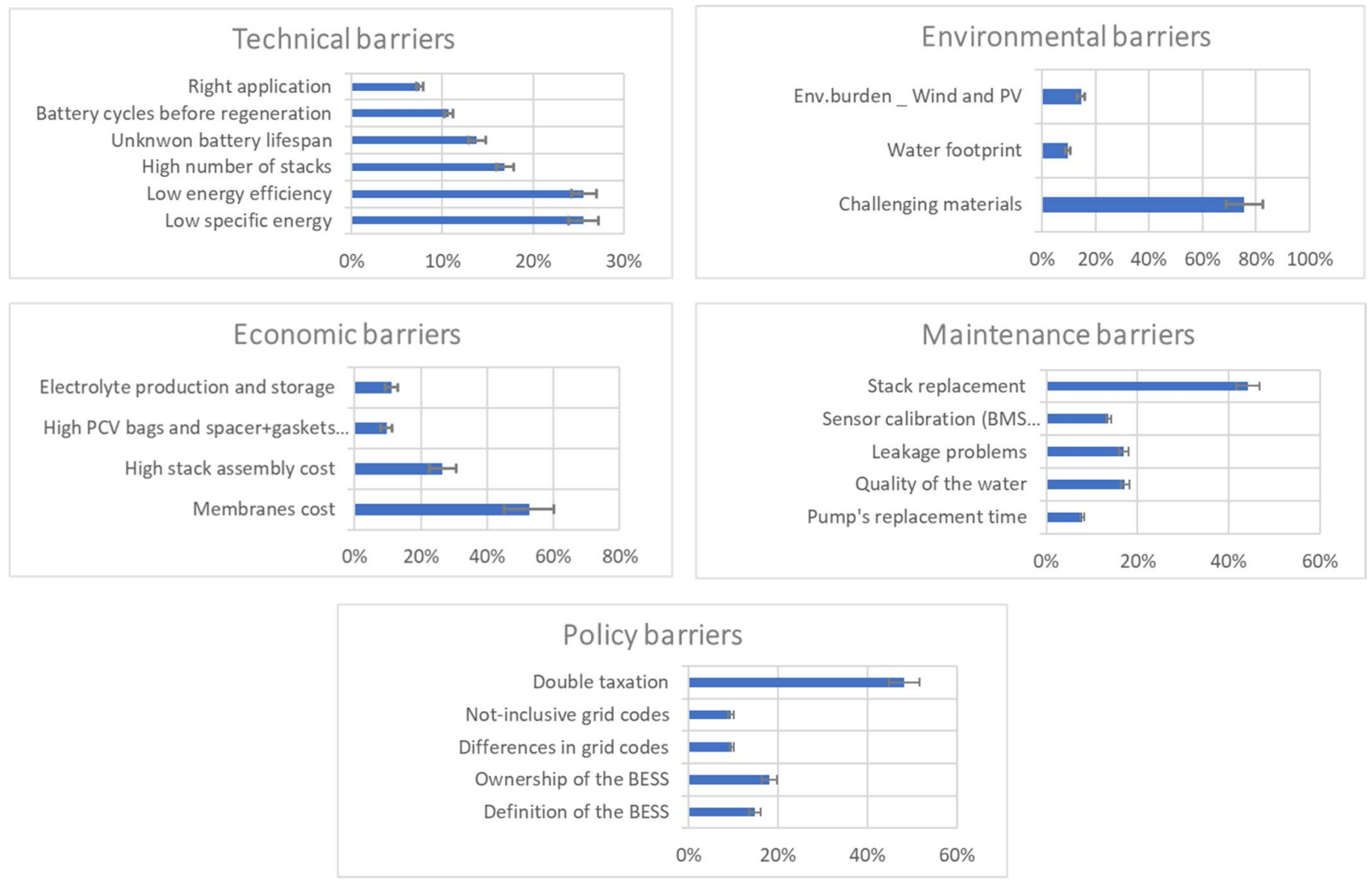

Figure 5. Local priorities for sub-criteria with respect to the goal.

Within the dominant economic criteria, the cost of the main battery components showed the most significant impacts. Specifically, the cost of the membranes was recognised by the experts as the main barrier, accounting for $65 \%$ of the stack costs. The relevance of the economic issues has also been reported in the literature [35]. Generally, the most significant barrier to ESS deployment is high capital costs. However, recent developments have indicated that capital costs are decreasing, and the ESS may be a preferred economic alternative. Furthermore, as assessed in Section 3.1.2, we expect a reduction in the membrane manufacturing costs in the long term via mass production for battery systems [36].

The second relevant category referred to the technical aspects. In this case, low specific energy and low energy round-trip efficiency (see Figure 5) showed the highest relevance. They were also recognised as important constraints of the ESS technologies by Kim et al. [30]. According to Section 3.1.2, these two technical characteristics represent a challenging condition to be overcome in the long term. Current battery research is focused on new materials and chemical compositions that will enhance the energy density in the future [36]. 
Environmental issues comprised the third category. Their relevance to the implementation of the new battery system has also been identified in the literature [21,30]. In terms of sustainability issues, the environment is an integral part of the three pillars, as seen in Figure 2. In this case, the use of challenging materials was the most important barrier among the environmental barriers (see Figure 5). Despite this, the AB-FB technology exhibited a low environmental impact, compared to the VRFB system, but steel and copper from the power and peripheral components increased the impact. Replacement of these materials with components based on recycled material fractions can be an option to decrease the environmental burden in future designs.

In terms of maintenance barriers, the stack replacement presented the main limitation to the implementation of the new technology. As described in Section 3.1.2, this component represents $65 \%$ of the total stack cost. For this reason, its replacement substantially increases the total maintenance cost of the system during its lifetime.

Regarding policies, as described in Section 3.1.2, it is evident that a further deployment of the ESS is limited by several market and regulatory barriers. Based on Figure 5, double taxation is the main restriction referred to in the policies. Although this barrier mostly affects local markets in Austria, Germany, Finland, and the Netherlands, it might negatively influence the ESS implementation roadmap.

Figure 6 shows the overall priorities of the 23 detected barriers or sub-criteria. The three sub-criteria with the highest global priority are membrane cost $(20 \%)$, challenging materials $(11 \%)$, and high stack assembly cost $(10 \%)$. These results highlighted that the most relevant barriers were related to economic and environmental aspects and confirmed the significant influence of the two main sustainability pillars on the implementation of the new technology.

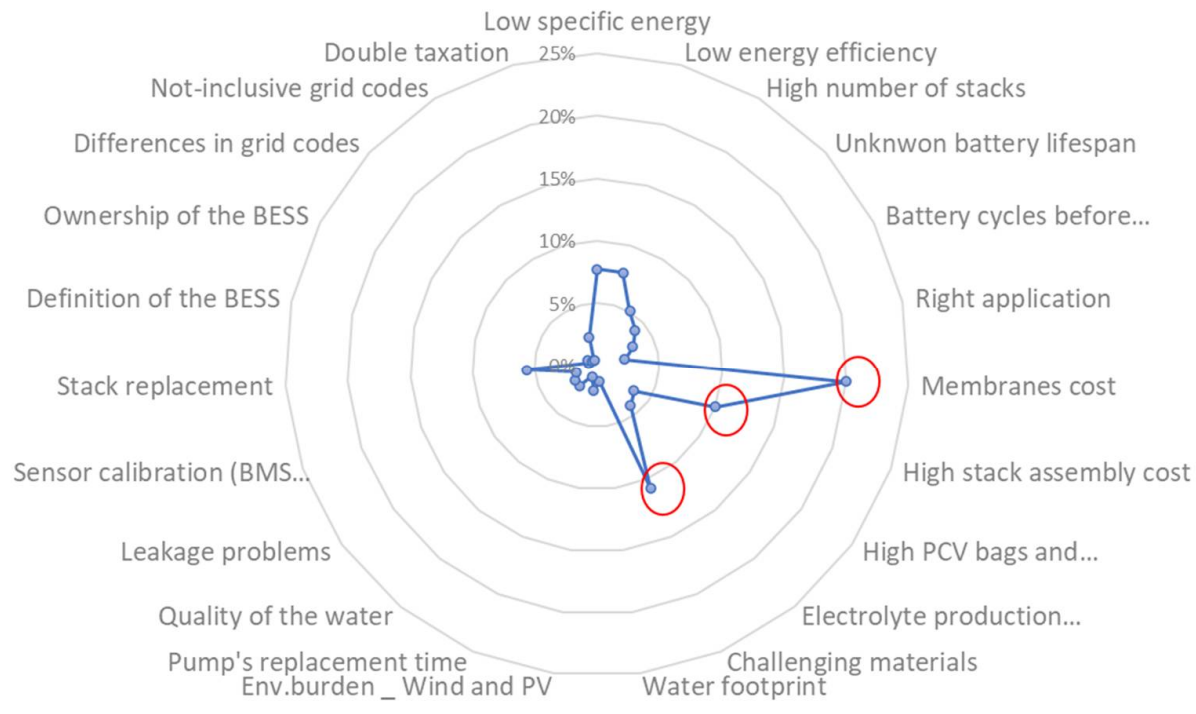

Figure 6. Overall priorities for sub-criteria with respect to the goal.

\section{Conclusions}

The AB-FB system technology constitutes a new approach to overcoming the supply intermittence linked to solar and wind power. Nevertheless, there are different barriers that might limit the implementation of this system for covering specific demands under a sustainable perspective. To elucidate the relevance of the possible barriers, we needed to carry out a deeper assessment of these conditioning parameters.

To advance the knowledge of these barriers, this work has, first, involved the revision of possible barriers related to five criteria: technical, economic, maintenance, environment, and policies. A second stage of the approach was focused on applying the AHP method to prioritise the identified barriers. Third, guidelines to overcome the related barrier effects were proposed. 
In this assessment, economic criteria achieved the highest-ranking position, followed by technical and environmental issues, in that order of relevance. Comparison of subcriteria determined that the highest global priority corresponded to the membrane cost (economic sub-criteria) followed by the challenging materials (environmental sub-criteria). This prioritisation confirmed the significant influence of the capital cost investment when a new ESS is deployed. This aspect can affect any ESS, albeit the sustainability of this AB-FB system, as well as its final cost, justifies its implementation.

The results attained in this work provide insights into which aspects should be addressed to make the battery even more sustainable and more affordable. This study contributes to identifying new research lines that should be pursued to improve the battery efficiency and to minimise energy losses during renewable support operation. Efforts should be directed towards finding innovative materials for the membranes and other battery components. A reduction in the consumption of materials such as steel and plastic will be a challenge. In this sense, circular economy strategies should be explored for the battery components by, for instance, considering the use of recycled materials.

Author Contributions: Conceptualisation, methodology, formal analysis, writing - original draft, writing-review and editing, M.C.D.-R. and M.B.-d.-P. All authors have read and agreed to the published version of the manuscript.

Funding: This work was performed within the framework of the BAoBaB project (Blue Acid/Base Battery: Storage and recovery of renewable electrical energy by reversible saltwater dissociation). The BAoBaB project received funding from the European Union's Horizon 2020 Research and Innovation programme under grant agreement no. 731187 (www.baobabproject.eu, accessed on 3 September 2021).

Acknowledgments: The authors express their gratitude to the BAoBaB project's partners from AquaBattery BV, Wetsus (European Centre of Excellence for Sustainable Water Technology), FUJIFILM (Fujifilm Manufacturing Europe BV), UNIPA (Universita Degli Studi di Palermo), and SMEDE (S.MED.E Pantelleria SPA) for their technical support in defining the barriers' implications and recommendations.

Conflicts of Interest: The authors declare no conflict of interest. The funders had no role in the design of the study; in the collection, analyses, or interpretation of data; in the writing of the manuscript; or in the decision to publish the results.

\section{References}

1. Díaz-Ramírez, M.C.; Ferreira, V.J.; García-Armingol, T.; López-Sabirón, A.M.; Ferreira, G. Environmental Assessment of Electrochemical Energy Storage Device Manufacturing to Identify Drivers for Attaining Goals of Sustainable Materials 4.0. Sustainability 2020, 12, 342. [CrossRef]

2. Sánchez-Díez, E.; Ventosa, E.; Guarnieri, M.; Trovò, A.; Flox, C.; Marcilla, R.; Soavi, F.; Mazur, P.; Aranzabe, E.; Ferret, R. Redox flow batteries: Status and perspective towards sustainable stationary energy storage. J. Power Sources 2021, 481, 228804. [CrossRef]

3. Pusceddu, E.; Zakeri, B.; Castagneto Gissey, G. Synergies between energy arbitrage and fast frequency response for battery energy storage systems. Appl. Energy 2021, 283, 116274. [CrossRef]

4. Keck, F.; Lenzen, M.; Vassallo, A.; Li, M. The impact of battery energy storage for renewable energy power grids in Australia. Energy 2019, 173, 647-657. [CrossRef]

5. Kebede, A.A.; Coosemans, T.; Messagie, M.; Jemal, T.; Behabtu, H.A.; Van Mierlo, J.; Berecibar, M. Techno-economic analysis of lithium-ion and lead-acid batteries in stationary energy storage application. J. Energy Storage 2021, 40, 102748. [CrossRef]

6. Mao, J.; Jafari, M.; Botterud, A. Planning low-carbon distributed power systems: Evaluating the role of energy storage. Energy 2022, 238, 121668. [CrossRef]

7. He, G.; Ciez, R.; Moutis, P.; Kar, S.; Whitacre, J.F. The economic end of life of electrochemical energy storage. Appl. Energy 2020, 273, 115151. [CrossRef]

8. Huang, C.; Xu, T. Electrodialysis with Bipolar Membranes for Sustainable Development. Environ. Sci. Technol. 2006, 40, 5233-5243. [CrossRef] [PubMed]

9. $\quad$ van Egmond, W.J.; Saakes, M.; Noor, I.; Porada, S.; Buisman, C.J.N.; Hamelers, H.V.M. Performance of an environmentally benign acid base flow battery at high energy density. Int. J. Energy Res. 2018, 42, 1524-1535. [CrossRef]

10. Pärnamäe, R.; Gurreri, L.; Post, J.; Johannes Van Egmond, W.; Culcasi, A.; Saakes, M.; Cen, J.; Goosen, E.; Tamburini, A.; Vermaas, D.A.; et al. The Acid-Base Flow Battery: Sustainable Energy Storage via Reversible Water Dissociation with Bipolar Membranes. Membranes 2020, 10, 409. [CrossRef] [PubMed] 
11. Culcasi, A.; Gurreri, L.; Zaffora, A.; Cosenza, A.; Tamburini, A.; Micale, G. On the modelling of an Acid/Base Flow Battery: An innovative electrical energy storage device based on $\mathrm{pH}$ and salinity gradients. Appl. Energy 2020, 277, 115576. [CrossRef]

12. Díaz-Ramírez, M.C.; Blecua-de-Pedro, M.; Arnal, A.J.; Post, J. Acid/Base Flow Battery Environmental and Economic Performance based on Its Potential Service to Renewables Support (Manuscript Submitted for Publication). 2021; Unpublished work.

13. European Commission. Critical Raw Materials Resilience: Charting a Path towards Greater Security and Sustainability; European Commission: Brussels, Belgium, 2020.

14. Gouveia, J.; Mendes, A.; Monteiro, R.; Mata, T.M.; Caetano, N.S.; Martins, A.A. Life cycle assessment of a vanadium flow battery. Energy Rep. 2020, 6, 95-101. [CrossRef]

15. Díaz-Ramírez, M.C.; Ferreira, V.J.; García-Armingol, T.; López-Sabirón, A.M.; Ferreira, G. Battery Manufacturing Resource Assessment to Minimise Component Production Environmental Impacts. Sustainability 2020, 12, 6840. [CrossRef]

16. Mardani, A.; Zavadskas, E.K.; Khalifah, Z.; Zakuan, N.; Jusoh, A.; Nor, K.M.; Khoshnoudi, M. A review of multi-criteria decision-making applications to solve energy management problems: Two decades from 1995 to 2015. Renew. Sustain. Energy Rev. 2017, 71, 216-256. [CrossRef]

17. Ribeiro, F.; Ferreira, P.; Araújo, M. Evaluating future scenarios for the power generation sector using a Multi-Criteria Decision Analysis (MCDA) tool: The Portuguese case. Energy 2013, 52, 126-136. [CrossRef]

18. Kurbatova, A.; Ahmed Abu-Qdais, H. Using Multi-Criteria Decision Analysis to Select Waste to Energy Technology for a Mega City: The Case of Moscow. Sustainability 2020, 12, 9828. [CrossRef]

19. Klein, S.J.W. Multi-Criteria Decision Analysis of Concentrated Solar Power with Thermal Energy Storage and Dry Cooling. Environ. Sci. Technol. 2013, 47, 13925-13933. [CrossRef]

20. Özkan, B.; Kaya, İ.; Cebeci, U.; Başlıgil, H. A Hybrid Multicriteria Decision Making Methodology Based on Type-2 Fuzzy Sets For Selection Among Energy Storage Alternatives. Int. J. Comput. Intell. Syst. 2015, 8, 914-927. [CrossRef]

21. Algarin, C.A.R.; Llanos, A.P.; Castro, A.O. An Analytic Hierarchy Process Based Approach for Evaluating Renewable Energy Sources. Int. J. Energy Econ. Policy 2017, 7, 38-47.

22. Culcasi, A.; Gurreri, L.; Micale, G.; Tamburini, A. Bipolar membrane reverse electrodialysis for the sustainable recovery of energy from $\mathrm{pH}$ gradients of industrial wastewater: Performance prediction by a validated process model. J. Environ. Manage. 2021, 287, 112319. [CrossRef]

23. Xia, J.; Eigenberger, G.; Strathmann, H.; Nieken, U. Acid-Base Flow Battery, Based on Reverse Electrodialysis with Bi-Polar Membranes: Stack Experiments. Processes 2020, 8, 99. [CrossRef]

24. Lim, A.; Lee, J.S.; Lee, S.; Lee, S.Y.; Kim, H.; Yoo, S.J.; Jang, J.H.; Sung, Y.-E.; Park, H.S. Polymer electrolyte membrane unitized regenerative fuel cells: Operational considerations for achieving high round trip efficiency at low catalyst loading. Appl. Catal. $B$ Environ. 2021, 297, 120458. [CrossRef]

25. Ubertini, S.; Facci, A.L.; Andreassi, L. Hybrid Hydrogen and Mechanical Distributed Energy Storage. Energies 2017, 10, 35. [CrossRef]

26. Weber, S.; Peters, J.F.; Baumann, M.; Weil, M. Life Cycle Assessment of a Vanadium Redox Flow Battery. Environ. Sci. Technol. 2018, 52, 10864-10873. [CrossRef] [PubMed]

27. Zaffora, A.; Culcasi, A.; Gurreri, L.; Cosenza, A.; Tamburini, A.; Santamaria, M.; Micale, G. Energy Harvesting by Waste Acid/Base Neutralization via Bipolar Membrane Reverse Electrodialysis. Energies 2020, 13, 5510. [CrossRef]

28. Tanaka, Y. 17-Bipolar Membrane Electrodialysis. In Ion Exchange Membranes, 2nd ed.; Tanaka, Y., Ed.; Elsevier: Amsterdam, the Netherlands, 2015; pp. 369-392. ISBN 978-0-444-63319-4.

29. Strathmann, H. (Ed.) Membrane Science and Technology Series. In Ion-Exchange Membrane Separation Processes; Membrane Science and Technology; Elsevier: Amsterdam, The Netherlands, 2004; Volume 9, p. ii.

30. Kim, J.; Suharto, Y.; Daim, T.U. Evaluation of Electrical Energy Storage (EES) technologies for renewable energy: A case from the US Pacific Northwest. J. Energy Storage 2017, 11, 25-54. [CrossRef]

31. Escalante Soberanis, M.A.; Mithrush, T.; Bassam, A.; Mérida, W. A sensitivity analysis to determine technical and economic feasibility of energy storage systems implementation: A flow battery case study. Renew. Energy 2018, 115, 547-557. [CrossRef]

32. Kapila, S.; Oni, A.O.; Kumar, A. The development of techno-economic models for large-scale energy storage systems. Energy 2017, 140, 656-672. [CrossRef]

33. Smallbone, A.; Jülch, V.; Wardle, R.; Roskilly, A.P. Levelised Cost of Storage for Pumped Heat Energy Storage in comparison with other energy storage technologies. Energy Convers. Manag. 2017, 152, 221-228. [CrossRef]

34. Huang, Y.; Chen, H.S.; Zhang, X.J.; Keatley, P.; Huang, M.J.; Vorushylo, I.; Wang, Y.D.; Hewitt, N.J. Techno-economic Modelling of Large Scale Compressed Air Energy Storage Systems. Energy Procedia 2017, 105, 4034-4039. [CrossRef]

35. Bhatnagar, D.; Currier, A.; Hernandez, J.; Ma, O.; Kirby, B. Market and Policy Barriers to Energy Storage Deployment A Study for the Energy Storage Systems Program; Sandia National Lab.: Albuquerque, NM, USA, 2013.

36. Schrøder Pedersen, A. Joint EASE/EERA Recommendations for a European Energy Storage Technology Development Roadmap towards 2030-Final; European Association for Storage of Energy (EASE): Brussels, Belgium, 2013. 